\title{
The Expansion Dance of Alpha Centauri
}

\author{
Dr. Andreas Gimsa
}

Stirling Technologie Institut gemeinnützige GmbH, D-14478 Potsdam, Germany

\begin{abstract}
Using Alpha Centauri as an example, it will be investigated how two-mass systems rotating around their barycenter evolve with the age of the universe. It is calculated how change the masses, the periods, the distance and the gravitational value. As the universe ages, both mases fall, the gravitational value increases quadratically, all system periods increase, and the distance between the two objects increases with the periods. The eccentric dance of the two stars expands accordingly.
\end{abstract}

Keywords :_Two-mass systems, Double stars, Alpha Centauri, Mass loss

Introduction

Two-mass systems rotating around a common center of mass are common in the universe. Usually, the two objects of such two-mass systems have different orbital velocities and they rotate in elliptical orbits around the common center of gravity. For example, the Earth-Moon system can be described as such a system. A high fraction of stars exists in galaxies in binary systems. Observation shows that even about $70 \%$ of all stars can be found in binary star systems. As an exemplary double star system Alpha Centauri shall be investigated here (Figure 1). The two fixed stars Alpha Centauri A and Alpha Centauri B are with a distance of 4.367 light-years closest to our Sun. Thus, they are quite well studied and can be studied further in the future. A similar exemplary calculation for a two-mass system rotating around its barycenter I had already carried out once [1.] and was guided thereby by the faulty assumption that gravitational waves propagating in space really exist and that the gravitational constant remains constant. However, these two assumptions are incorrect from my today's point of view (see also point 4.).

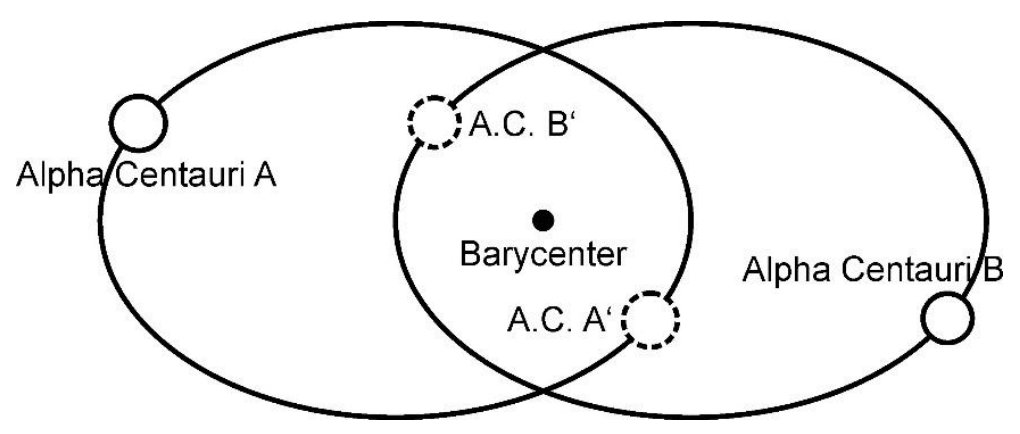

Figure 1: $\quad$ Model sketch of Alpha Centauri (without scale) 
Independently of this, the principle of the constant number of effects would have to be valid in the universe. Only with recognition of this principle the unification of gravitation with quantum physics succeeds. A consequence of this principle is that the product of age and mass of the universe must remain constant. All masses of the universe must fall with the time, so that the principle of the constant number of the effects $N$ is preserved. The entropy equation (01) can be interpreted as the law of conservation of information: The law of conservation of potential information, for which entropy stands. Every point with the distance from the radius of the expanding spherical universe to its center moves exactly with the speed of light. Therefore, the universe can be considered as a closed and completely thermally isolated system undergoing an isentropic change of state. The equation for its invariable entropy is valid for the universe [2.]:

$$
S_{\text {uni }}=\frac{k}{\hbar} m_{\text {uni }} t_{\text {uni }} c^{2}=\text { const. }
$$

with $k$ as Boltzmann constant and $\hbar=h /(2 \pi)$ as the reduced Planck constant. For the term $m_{\text {uni }} t_{\text {uni }} c^{2}$ can also be written $N \hbar$, a constant number of effects during the aging of the universe. The mass of the universe $m_{u n i}$ and with it all object masses must fall with age, since all other coefficients in equation (01) remain constant.

\section{Object of investigation}

Because the double star system Alpha Centauri as a fixed star system offers the best observing possibilities after the sun because of its proximity to the earth, it shall be investigated concerning important parameters. These are the evolution of the masses, the proper rotation periods of both stars, their distance, the gravitational constant and the angular momentum. For the system Earth-Moon a similar consideration was already accomplished, which considered there the tidal influence [3.]. Tidal effects, the influence of further masses and mass transport mechanisms between both stars are regarded as negligible here and are not considered.

The following parameters are taken as given (all values in SI units):

\section{Alpha Centauri A $\quad \underline{\text { Alpha Centauri B }}$}

$\begin{array}{llll}\mathrm{m}[\mathrm{kg}] & 2.19775710^{30} & 1.85765110^{30} & \text { (masses are variable) } \\ \mathrm{T}_{\mathrm{A} / \mathrm{B}}[\mathrm{s}] & 1.90080010^{06} & 3.54240010^{06} & \text { (Intrinsic rotations are variable) }\end{array}$

\section{Common parameters}

$\begin{array}{lll}\mathrm{c}[\mathrm{m} / \mathrm{s}] & 2.99790010^{08} & \text { (speed of light is constant) } \\ \gamma\left[\mathrm{m}^{3} /\left(\mathrm{kg} \mathrm{s}^{2}\right)\right] & 6.67430010^{-11} & \text { (gravitational value is variable) } \\ \mathrm{t}_{\text {uni }}[\mathrm{s}] & 4.35196810^{17} & \text { (age of the universe is variable) } \\ \mathrm{T}[\mathrm{s}] & 2.51972610^{09} & \text { (common orbital period is variable) } \\ \mathrm{e} & 5.19000010^{-01} & \text { (eccentricity is constant) } \\ \mathrm{T}_{\text {step }}[\mathrm{s}] & 3.15360010^{15} & \text { (step size is variable, } \mathrm{T}_{\text {step } 0}=100 \text { million years) }\end{array}$

The radii of both Alpha Centauri stars are small in relation to their distance. The ratio is about 1/10,000. Therefore the stars can be considered as point masses with respect to their common orbital motion.

The radiation-induced mass loss of the Sun is about $1.310^{14} \mathrm{~kg} / \mathrm{a}$. The mass loss resulting from the radiation of the two stars of Alpha Centauri is in the same order of magnitude. Quite different is the mass loss, which results from the mass decay. It is $1.610^{20} \mathrm{~kg} / \mathrm{a}$ for Alpha Centauri A and $1.310^{20} \mathrm{~kg} / \mathrm{a}$ for Alpha Centauri B (see point 2. Calculation basis). The mass loss due to decay is therefore 6 orders of magnitude larger than the mass loss due to radiation, which can therefore be neglected. 


\section{Calculation bases}

\begin{tabular}{|c|c|c|c|c|c|c|c|c|}
\hline & tuni & tstep & $\mathbf{T}$ & $\mathbf{\Delta T}$ & $\mathbf{T}_{\mathbf{A}}$ & $\mathbf{T}_{\mathbf{B}}$ & $\gamma$ & $\mathbf{r}$ \\
\hline $\mathbf{1 .}$ & $4.351968 \mathrm{E}+17$ & $3.153600 \mathrm{E}+15$ & $2.519726 \mathrm{E}+09$ & $0.000000 \mathrm{E}+00$ & $1.900800 \mathrm{E}+06$ & $3.542400 \mathrm{E}+06$ & $6.674300 \mathrm{E}-11$ & $3.517729 \mathrm{E}+12$ \\
\hline $\mathbf{2 .}$ & $4.383504 \mathrm{E}+17$ & $3.176452 \mathrm{E}+15$ & $2.537985 \mathrm{E}+09$ & $1.825889 \mathrm{E}+07$ & $1.914574 \mathrm{E}+06$ & $3.568070 \mathrm{E}+06$ & $6.771379 \mathrm{E}-11$ & $3.543220 \mathrm{E}+12$ \\
\hline $\mathbf{3 .}$ & $4.415269 \mathrm{E}+17$ & $3.199470 \mathrm{E}+15$ & $2.556376 \mathrm{E}+09$ & $1.839120 \mathrm{E}+07$ & $1.928448 \mathrm{E}+06$ & $3.593925 \mathrm{E}+06$ & $6.869871 \mathrm{E}-11$ & $3.568896 \mathrm{E}+12$ \\
\hline $\mathbf{4 .}$ & $4.447263 \mathrm{E}+17$ & $3.222655 \mathrm{E}+15$ & $2.574901 \mathrm{E}+09$ & $1.852447 \mathrm{E}+07$ & $1.942422 \mathrm{E}+06$ & $3.619968 \mathrm{E}+06$ & $6.969795 \mathrm{E}-11$ & $3.594757 \mathrm{E}+12$ \\
\hline $\mathbf{5 .}$ & $4.479490 \mathrm{E}+17$ & $3.246007 \mathrm{E}+15$ & $2.593560 \mathrm{E}+09$ & $1.865870 \mathrm{E}+07$ & $1.956497 \mathrm{E}+06$ & $3.646200 \mathrm{E}+06$ & $7.071173 \mathrm{E}-11$ & $3.620806 \mathrm{E}+12$ \\
\hline $\mathbf{6 .}$ & $4.511950 \mathrm{E}+17$ & $3.269529 \mathrm{E}+15$ & $2.612354 \mathrm{E}+09$ & $1.879391 \mathrm{E}+07$ & $1.970675 \mathrm{E}+06$ & $3.672621 \mathrm{E}+06$ & $7.174025 \mathrm{E}-11$ & $3.647044 \mathrm{E}+12$ \\
\hline $\mathbf{7 .}$ & $4.544645 \mathrm{E}+17$ & $3.293221 \mathrm{E}+15$ & $2.631284 \mathrm{E}+09$ & $1.893010 \mathrm{E}+07$ & $1.984955 \mathrm{E}+06$ & $3.699235 \mathrm{E}+06$ & $7.278373 \mathrm{E}-11$ & $3.673472 \mathrm{E}+12$ \\
\hline $\mathbf{8 .}$ & $4.577577 \mathrm{E}+17$ & $3.317085 \mathrm{E}+15$ & $2.650351 \mathrm{E}+09$ & $1.906727 \mathrm{E}+07$ & $1.999339 \mathrm{E}+06$ & $3.726041 \mathrm{E}+06$ & $7.384239 \mathrm{E}-11$ & $3.700091 \mathrm{E}+12$ \\
\hline $\mathbf{9 .}$ & $4.610748 \mathrm{E}+17$ & $3.341122 \mathrm{E}+15$ & $2.669556 \mathrm{E}+09$ & $1.920544 \mathrm{E}+07$ & $2.013827 \mathrm{E}+06$ & $3.753041 \mathrm{E}+06$ & $7.491644 \mathrm{E}-11$ & $3.726903 \mathrm{E}+12$ \\
\hline $\mathbf{1 0 .}$ & $4.644159 \mathrm{E}+17$ & $3.365333 \mathrm{E}+15$ & $2.688901 \mathrm{E}+09$ & $1.934461 \mathrm{E}+07$ & $2.028420 \mathrm{E}+06$ & $3.780237 \mathrm{E}+06$ & $7.600612 \mathrm{E}-11$ & $3.753910 \mathrm{E}+12$ \\
\hline
\end{tabular}

\begin{tabular}{|c|c|c|c|c|c|c|c|c|}
\hline & $\mathbf{\Delta r}$ & $\mathbf{r}_{\max }$ & $\mathbf{r}_{\mathbf{m i n}}$ & $\mathbf{m}_{\mathbf{A}}$ & $\mathbf{m}_{\mathbf{B}}$ & $\mathbf{\Delta} \mathbf{m}_{\mathrm{A}+\mathrm{B}}$ & Lorbit & Lorbit test \\
\hline $\mathbf{1 .}$ & $0.000000 \mathrm{E}+00$ & $5.343431 \mathrm{E}+12$ & $1.692028 \mathrm{E}+12$ & $2.197757 \mathrm{E}+30$ & $1.857651 \mathrm{E}+30$ & $0.000000 \mathrm{E}+00$ & $3.106423 \mathrm{E}+46$ & $3.106423 \mathrm{E}+46$ \\
\hline $\mathbf{2 .}$ & $2.549079 \mathrm{E}+10$ & $5.382152 \mathrm{E}+12$ & $1.704289 \mathrm{E}+12$ & $2.181945 \mathrm{E}+30$ & $1.844287 \mathrm{E}+30$ & $2.917560 \mathrm{E}+28$ & $3.106423 \mathrm{E}+46$ & $3.106423 \mathrm{E}+46$ \\
\hline $\mathbf{3 .}$ & $2.567551 \mathrm{E}+10$ & $5.421153 \mathrm{E}+12$ & $1.716639 \mathrm{E}+12$ & $2.166248 \mathrm{E}+30$ & $1.831019 \mathrm{E}+30$ & $2.896570 \mathrm{E}+28$ & $3.106423 \mathrm{E}+46$ & $3.106423 \mathrm{E}+46$ \\
\hline $\mathbf{4 .}$ & $2.586156 \mathrm{E}+10$ & $5.460436 \mathrm{E}+12$ & $1.729078 \mathrm{E}+12$ & $2.150663 \mathrm{E}+30$ & $1.817846 \mathrm{E}+30$ & $2.875731 \mathrm{E}+28$ & $3.106423 \mathrm{E}+46$ & $3.106423 \mathrm{E}+46$ \\
\hline $\mathbf{5 .}$ & $2.604897 \mathrm{E}+10$ & $5.500005 \mathrm{E}+12$ & $1.741608 \mathrm{E}+12$ & $2.135191 \mathrm{E}+30$ & $1.804768 \mathrm{E}+30$ & $2.855043 \mathrm{E}+28$ & $3.106423 \mathrm{E}+46$ & $3.106423 \mathrm{E}+46$ \\
\hline $\mathbf{6 .}$ & $2.623773 \mathrm{E}+10$ & $5.539860 \mathrm{E}+12$ & $1.754228 \mathrm{E}+12$ & $2.119830 \mathrm{E}+30$ & $1.791784 \mathrm{E}+30$ & $2.834503 \mathrm{E}+28$ & $3.106423 \mathrm{E}+46$ & $3.106423 \mathrm{E}+46$ \\
\hline $\mathbf{7 .}$ & $2.642786 \mathrm{E}+10$ & $5.580004 \mathrm{E}+12$ & $1.766940 \mathrm{E}+12$ & $2.104579 \mathrm{E}+30$ & $1.778893 \mathrm{E}+30$ & $2.814111 \mathrm{E}+28$ & $3.106423 \mathrm{E}+46$ & $3.106423 \mathrm{E}+46$ \\
\hline $\mathbf{8 .}$ & $2.661936 \mathrm{E}+10$ & $5.620439 \mathrm{E}+12$ & $1.779744 \mathrm{E}+12$ & $2.089439 \mathrm{E}+30$ & $1.766096 \mathrm{E}+30$ & $2.793865 \mathrm{E}+28$ & $3.106423 \mathrm{E}+46$ & $3.106423 \mathrm{E}+46$ \\
\hline $\mathbf{9 .}$ & $2.681226 \mathrm{E}+10$ & $5.661166 \mathrm{E}+12$ & $1.792641 \mathrm{E}+12$ & $2.074407 \mathrm{E}+30$ & $1.753390 \mathrm{E}+30$ & $2.773766 \mathrm{E}+28$ & $3.106423 \mathrm{E}+46$ & $3.106423 \mathrm{E}+46$ \\
\hline $\mathbf{1 0 .}$ & $2.700655 \mathrm{E}+10$ & $5.702189 \mathrm{E}+12$ & $1.805631 \mathrm{E}+12$ & $2.059483 \mathrm{E}+30$ & $1.740776 \mathrm{E}+30$ & $2.753810 \mathrm{E}+28$ & $3.106423 \mathrm{E}+46$ & $3.106423 \mathrm{E}+46$ \\
\hline
\end{tabular}

Table 1: Evolution of Alpha Centauri with the Age of the Universe

The description and formulae of the investigated parameters of the double star system are given in the order of the table columns of Table 1 .

The space age $t_{u n i}$ is currently 13.8 billion years. This corresponds to about $4.3510^{17}$ seconds. The space age grows in table 1 with each line by the step size $t_{\text {step }}$. The step size $t_{\text {step }}$ is the period, which is determined for the consideration of the change of parameters. It is 100 million years. This corresponds to $3.1510^{15}$ seconds. Since the table has 10 rows, a total change period of about 900 million years in the future is examined. The step size is not kept constant with the small change of all natural periods. It increases slightly.

The orbital period $T$ is the period of both masses during the orbit around the barycenter. It amounts to 79.9 years according to the observations. That corresponds to $2.5210^{09}$ seconds. With the age all natural periods increase and the energy levels reduce.

The temporality of the universe permeates all objects and processes. The simple formula for the determination of a future period is valid $T$ [4.]:

$$
T=T_{0}\left(1+\frac{\Delta t}{t_{u n i}}\right)
$$

Where $T_{0}$ is the current period and $\Delta t$ is the respective period under consideration. It is easy to see that, for example, the period change in one year must be very small.

The period change $\Delta T$ refers to a step size $t_{\text {step }}$. For example, the first period change is the difference of the period after 100 million years (2nd row of the table) and the 1st period (1st row of the table).

The intrinsic rotation periods $T_{A}$ and $T_{B}$ describe the rotation of both stars around their own axes. Equation (02) is also valid for these periods. With the space age the periods become larger. 
The gravitational value $\gamma$ is variable with the age of the universe. This can be understood best if one recognizes that for the preservation of the cosmic structure the ratio of electric force and gravitational force of proton and electron must always remain constant:

$$
\frac{F_{e l}}{F_{\text {grav }}}=\frac{e^{2} /\left(4 \pi \varepsilon_{0} r^{2}\right)}{\gamma m_{p r} m_{e l} / r^{2}}=\frac{e^{2} /\left(4 \pi \varepsilon_{0}\right)}{\gamma m_{p r} m_{e l}}=\text { const }
$$

Since the electric elementary charge and the electric field constant are constant and each mass falls with the age of the universe according to equation (01), the gravitational value must increase quadratically [5.]:

$$
\gamma=\gamma_{1}\left(1+\frac{\Delta t}{t_{u n i}}\right)^{2}
$$

As the age of the universe doubles, the gravitational value quadruples.

The mean distance $r$ of both stars results from the known formula for the common period:

$$
T=\sqrt{\frac{4 \pi^{2} r^{3}}{\gamma\left(m_{1}+m_{2}\right)}} \quad r=\sqrt[3]{\frac{T^{2} \gamma\left(m_{1}+m_{2}\right)}{4 \pi^{2}}}
$$

The equation for the change of the mean distance $r$ is [6.]:

$$
r=r_{0}\left(1+\frac{\Delta t}{t_{u n i}}\right)
$$

One could, because of the different powers of $r$ and $T$ in equation (05), suspect that equations (02) and (06) are in contradiction. The resolution is found in the quadratic increase of the gravitational value $\gamma$ according to equation (04). The cosmic clockwork works perfectly.

Due to the high eccentricity the actual distance of both stars varies strongly during each orbit. During their approach both velocities increase. The mean distance change $\Delta r$ refers just like the period change to a step size of $t_{\text {step }}$. For example, the first mean distance change is the difference of the mean distance change after 100 million years ( 2 nd row of the table) and the first mean distance change (1st row of Table 1).

The maximum distance $r_{\max }$ and the minimum distance $r_{\min }$ between the two stars are linked by the eccentricity $e$ with each other. It is known to hold:

$$
e=\frac{r_{\max }-r_{\min }}{r_{\max }+r_{\min }}
$$

Moreover, for the mean distance $r$ between the two stars:

$$
r=\frac{r_{\max }+r_{\min }}{2}
$$

From equations (07) and (08) it is possible to calculate from the mean distance $r$ and the eccentricity $e$ the maximum and minimum distance:

$$
r_{\text {max }}=r(1+e) \quad r_{\min }=r(1-e)
$$

As far as the temporal evolution is concerned, the distances increase $r_{\max }$ and $r_{\min }$ increase to the same extent as the mean distance $r$ according to equation (06).

The masses of both stars $m_{A 0}$ and $m_{B 0}$ at the present time are known. They can be found in the first row of table 1 . The masses decrease with the age of the universe according to the following formula [7.]: 


$$
m_{A}=\frac{m_{A 0}}{1+\frac{\Delta t}{t_{\text {uni }}}} \quad m_{B}=\frac{m_{B 0}}{1+\frac{\Delta t}{t_{\text {uni }}}}
$$

The mass change of both stars $\Delta m_{A+B}$ is calculated from row to row in Table 1, each for one step size.

The orbital angular momentum $L_{\text {orbit }}$ of both stars results from the sum of both angular momentums of the motion around the common center of gravity. For the mean center of gravity distances $r_{1}$ and $r_{2}$ of both masses can be written with a plausible assumption of point masses (see point 1.):

$$
L_{\text {orbit }}=L_{A}+L_{B}=m_{A} \overline{v_{A}} r_{A}+m_{B} \overline{v_{B}} r_{B}
$$

The center of gravity distances of both stars $r_{A}$ and $r_{B}$ are calculated as follows:

$$
r_{A}=\frac{m_{B}}{\left(m_{A}+m_{B}\right)} r \quad r_{B}=\frac{m_{A}}{\left(m_{A}+m_{B}\right)} r
$$

Moreover, both mean orbital velocities of the stars around the barycenter can be written with common period:

$$
\overline{v_{A}}=\frac{2 \pi r_{A}}{T}=\frac{r_{A}}{t} \quad \overline{v_{B}}=\frac{2 \pi r_{B}}{T}=\frac{r_{B}}{t}
$$

From equations (11), (12) and (13) it follows:

$$
L_{\text {orbit }}=m_{A} \frac{r_{A}^{2}}{t}+m_{B} \frac{r_{B}^{2}}{t}=m_{A} \frac{m_{B}^{2}}{\left(m_{A}+m_{B}\right)^{2}} \frac{r^{2}}{t}+m_{B} \frac{m_{A}{ }^{2}}{\left(m_{A}+m_{B}\right)^{2}} \frac{r^{2}}{t}
$$

The simplification leads to the common orbital angular momentum $L_{\text {orbit }}$ :

$$
L_{\text {orbit }}=\frac{m_{A} m_{B}}{m_{A}+m_{B}} \frac{r^{2}}{t} \quad \text { with } \quad t=\frac{T}{2 \pi}
$$

The orbital angular momentum of both masses remains. It does not change with increasing age of the universe!

As a check, another calculation was performed for the orbital angular momentum with the mean total velocity $\bar{v}=r / t=\overline{v_{A}}+\overline{v_{B}}, r=r_{A}+r_{B}$ and equation (05):

$$
L_{\text {orbit test }}=\frac{\gamma m_{A} m_{B}}{\bar{v}}=\gamma m_{A} m_{B} \frac{t}{r}
$$

Equation (16) gives the same result as equation (15) and is just as valid for the rotating described two-mass system (see Table 1). Also with the equations (15) and (16) one can realize that with constant angular momentum and the other variable quantities, the gravitational value $\gamma$ must increase quadratically with the space age.

The mean relativistic orbital velocities were not listed in table 1. However, it is interesting to know that also these velocities must remain absolutely constant. This is evident from the formula (18). The calculation formula for these velocities was already derived in source [8.]. The velocity of light of the objects cannot be exceeded at the orbit around the barycenter.

The factor 2 in the term of the denominator of equation (18) is astonishing. This comes from the double kinetic energy during the rotation. The following formula is valid for the rotation of a two mass system around its barycenter with the relativistic kinetic energy of both masses, which must be spent to separate them from their center distance to infinity: 


$$
E_{\text {grav }}=\int_{s=r}^{s=\infty} F_{\text {grav }} d s=\gamma m_{A} m_{B} \int_{s=r}^{s=\infty} \frac{1}{s^{2}} d s=\gamma \frac{m_{A} m_{B}}{r}=2\left(E_{\text {kin } A}+E_{\text {kin } B}\right)
$$

Without the factor 2 in equations (17) and (18) the orbital angular momentum according to equation (15) would not be able to be calculated corretly. During the translation of the free fall the kinetic energy of each mass does not occur twice, but only once [9.].

It is interesting to note in equation (17) that the term $\gamma m_{A} m_{B}$ can be placed as a constant in front of the integral. If, for example, the masses would decrease with increasing mean distance $r$ and the gravitational value $\gamma$ would remain constant, this integration would not be correct according to Newton.

$$
\overline{v_{\mathrm{A}}}=c \sqrt{1-\frac{1}{\left[\frac{\gamma m_{\mathrm{B}}^{2}}{2 c^{2} r\left(m_{\mathrm{A}}+m_{B}\right)}+1\right]^{2}}} \quad \overline{v_{\mathrm{B}}}=c \sqrt{1-\frac{1}{\left[\frac{\gamma m_{\mathrm{A}}^{2}}{2 c^{2} r\left(m_{\mathrm{A}}+m_{B}\right)}+1\right]^{2}}}
$$

\section{Evaluation of the calculation}

The calculation results according to the described formulas are summarized in table 1 . The total period of consideration with 10 steps amounts to approx. 900 million years.

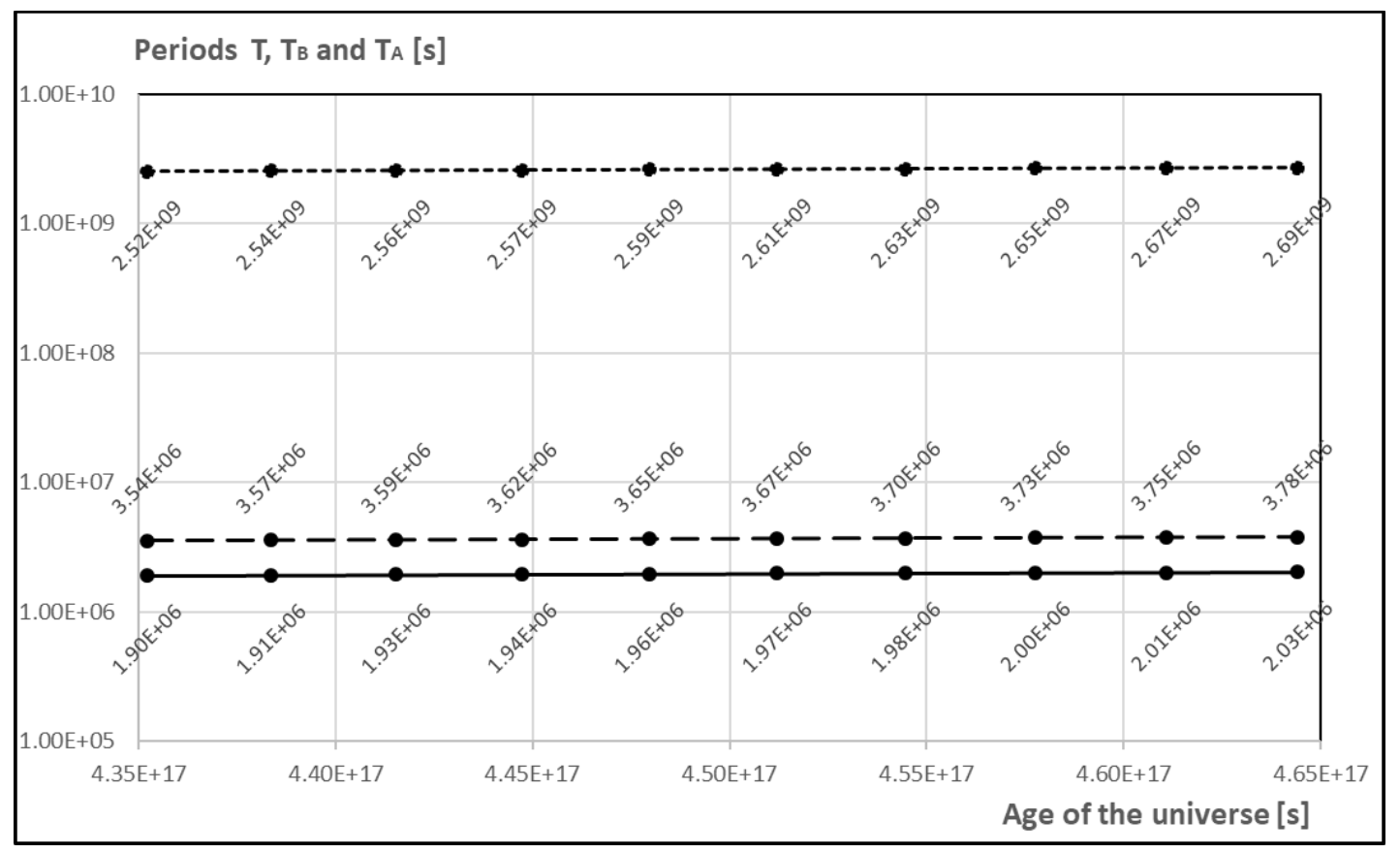

Diagram 1: The evolution of the periods with the age of the universe

The numbers in the diagram show that the periods increase proportionally with the age of the world. In order to show all periods in one diagram, a logarithmic scale was chosen, which however suggests that the periods remain constant. 


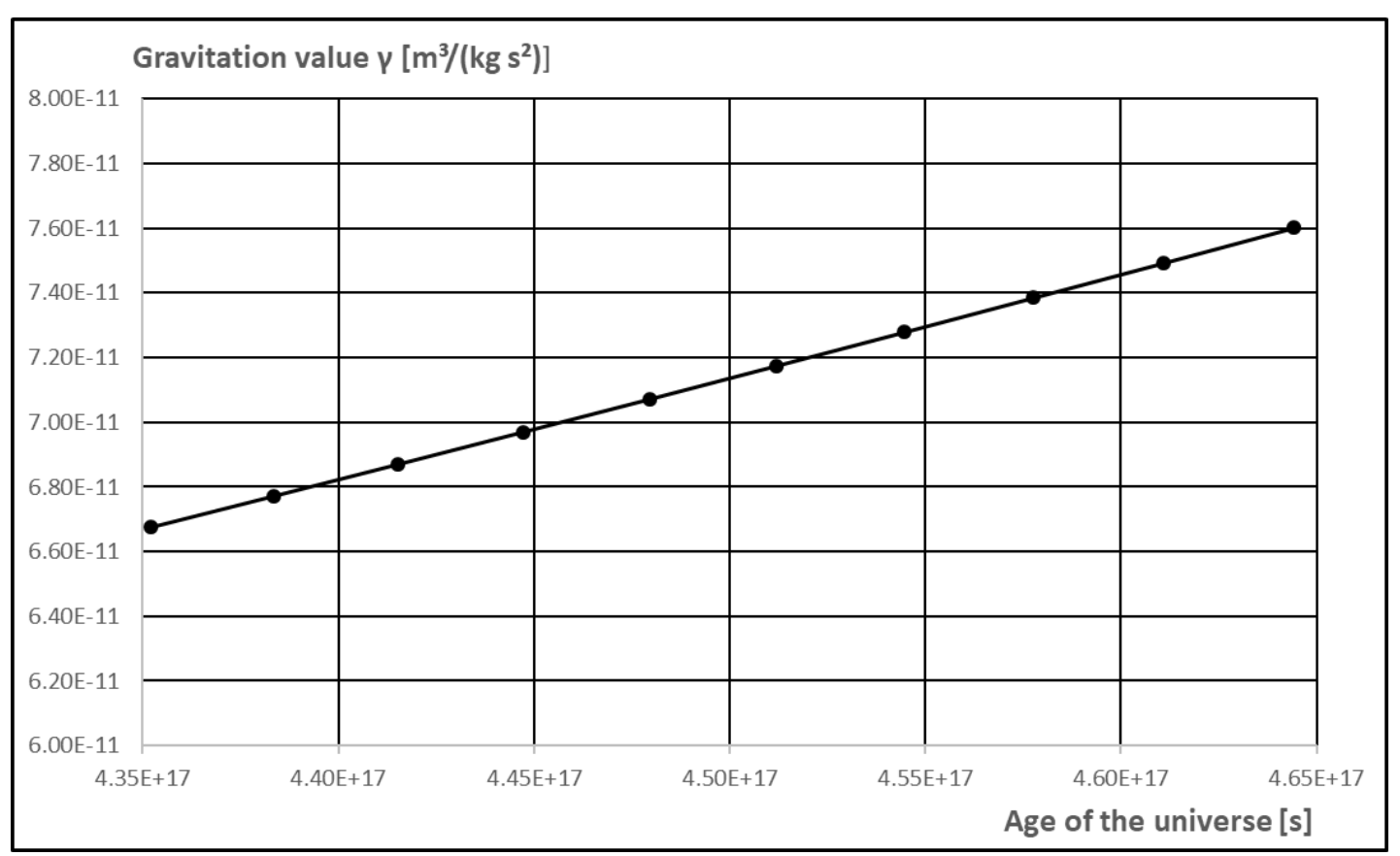

Diagram 2: The evolution of the gravitational value with the age of the universe

One recognizes in diagram 2 the quadratically growth of the gravity value $\gamma$ with the age of the universe. Since only a time interval of 900 million years in total is considered, the quadratic growth is not visible [10.].

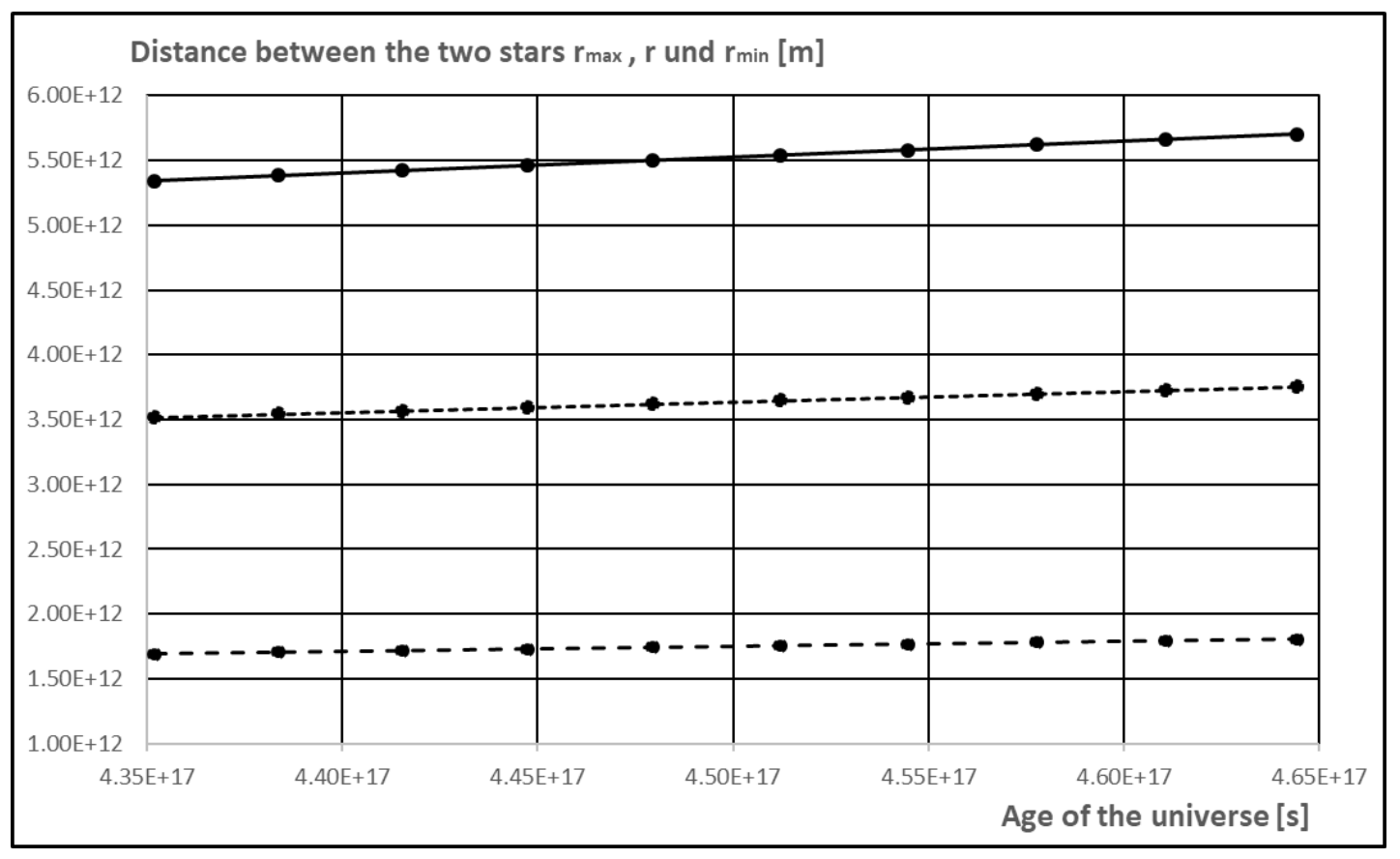

Diagram 3: The evolution of distances with the age of the universe

Just like the periods, the distances grow proportionally with the space age. This is an important realization, which must be absolutely considered at the expansion of the universe. It is to be noted that all earthly scales grow with it. 


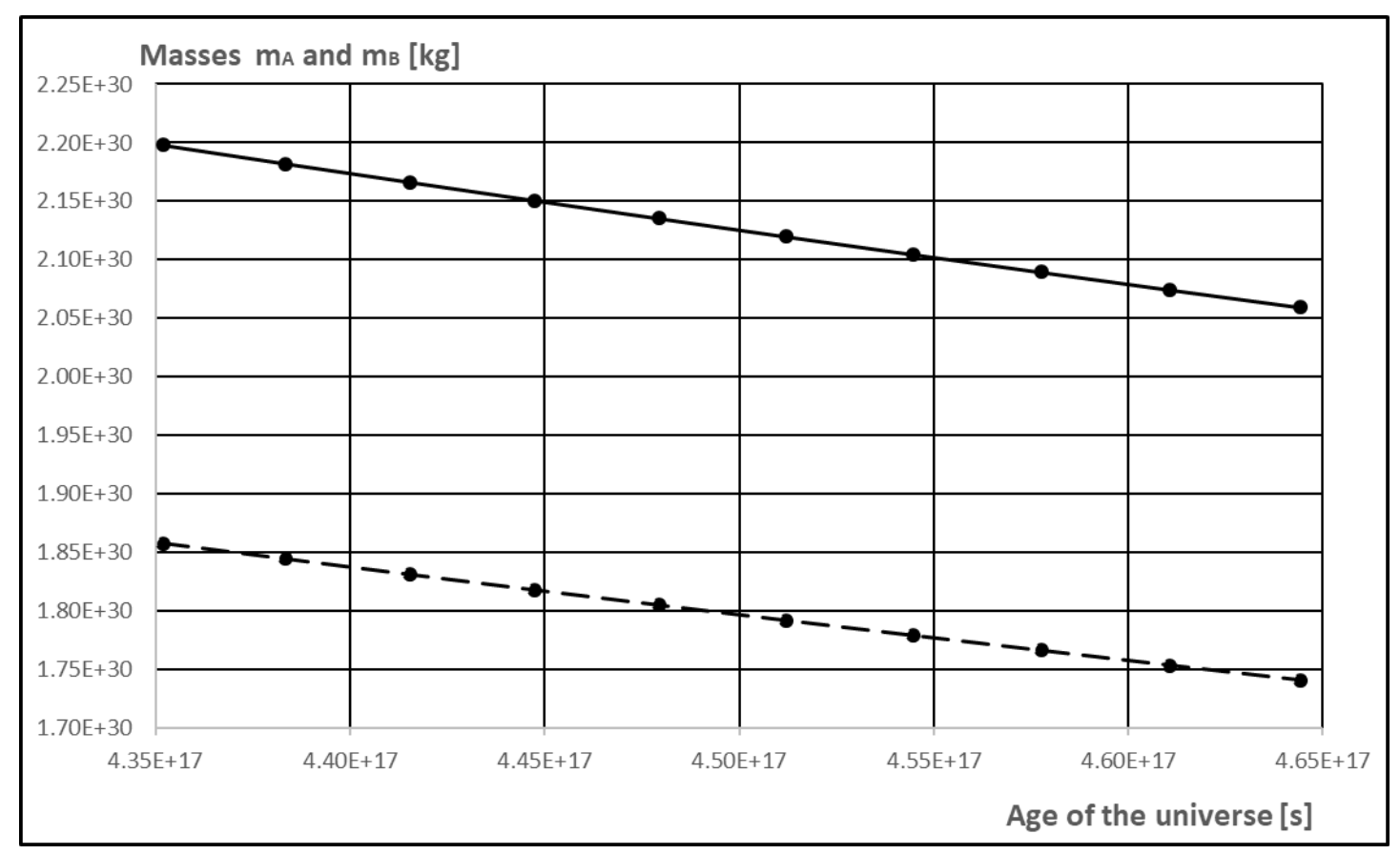

Diagram 4:-The development of the object masses with the age of the universe

The two masses fall with the age of the universe according to the formula (10), which could be derived from the entropy formula (01)

\section{Gravitational waves and mass decay}

Gravitational waves would have to be longitudinal waves contrary to the assumption of quadrupole waves and would not propagate in space but in the time dimension. Their direction of oscillation and propagation would then coincide. The propagation could take place via imaginary photons only in a second imaginary temporal dimension. These special photons could cause the interaction of magnetic monopoles $p$ which form the time only after the universal mass decay. The actual gravitational force in space can be described by the magnetic force of monopoles. For example, the gravitational force between proton and electron is: $\gamma m_{p} m_{e} / r^{2}=x p^{2} /\left(4 \pi \mu_{0} r^{2}\right)$. The attenuation in space is $x=r_{p} /\left(2 \alpha r_{u n i}\right)$ with the proton radius $r_{p}$, the radius of the universe $r_{u n i}$ and the fine structure constant $\alpha$.

A physical explanation for the phenomenon "time" has not been found so far, it is simply taken for granted today. All masses could consist of neutrinos in their smallest structure (e.g. the quarks and electrons). Magnetic monopoles, which could originate only from neutrinos at the decay, are possibly not encountered in the space, because they are time formers. A neutral object can be neutral only if it consists of balanced charges.

An electron neutrino could in this sense exists as a magnetic north pole and a magnetic south pole. The propagation of gravity via imaginary light with the velocity $c_{i}$ would allow instantaneous gravitational propagation. A description of gravitation, which is correct according to these criteria, which is compatible to quantum physics, and finally leads to the Newtonian description, I have made in source [11.].

\section{Shot conclusions}

With the double star system Alpha Centauri A and B an exemplary calculation was made, whose changing parameters must be representative for the whole universe. Because of the operation of atomic clocks [12.] it should be possible to calculate the periods increasing from year to year from the beginning to measure: $T_{A 0}=22 d$ and $T_{B 0}=41 d$. If one sets in the calculation the step size $t_{\text {step }}$ to one year, one receives the 
parameter changes after one year with the described calculation formulas. The common orbital period $T$ becomes 0.18 seconds larger in the year.

In [13.] I have calculated for the system Earth-Moon from the Earth-own angular momentum $L_{1}$ and the common orbital angular momentum $L_{2}$ that the observed annual lengthening of a day of $16.5 \mu$ s has 2 causes: 1. a lengthening of $10.2 \mu$ s by tidal friction and 2 . a lengthening of $6.3 \mu$ s by the enlargement of all periods according to equation (02).

The mean distance $r$ between both stars grows by 255 meters per year and the mass loss $\Delta m_{A+B}$ of both stars in one year is $2.9410^{20} \mathrm{~kg}$.

The computational evidence shows us the following: In the eccentric dance of both stars, both their distance and period increase and their masses decrease. The gravitational value increases and the mean orbital velocities and the orbital angular momentum remain constant.

These results are immensely important and necessary for a better understanding of the world, with which a unification of quantum physics and gravitation can only succeed. For this, the recognition of equation (01) is indispensable.

\section{References}

Since these are novel physical findings discovered, researched, theoretically described and proven by me, I am dependent on relying primarily on my own sources.

[1.] Gimsa, A., Dual mass systems under the influence of gravitation, published by the author, Potsdam, Germany, 2017, ISBN 978-3-00-057218-0, p.25

[2.] Gimsa, A., The metric of space-time, English-German 2nd edition, published by the author, Potsdam, Germany 2020, ISBN 978-3-00-064784-0, p.26

[3.] Gimsa, A., Development of the Distance Earth-Moon, International Journal of Scientific Research a. M., Volume 08, Issue 03, March 2020, DOI: 10.18535/ijsrm/v8i03.aa01

[4.] See Source [2.], p.39

[5.] Gimsa, A., The beauty of nature, Gieselmann Druck- und Medienhaus, Potsdam, Germany, 2014, ISBN 978-3-923830-94-7, p.59

[6.] See Source [2.], p.38

[7.] See Source [2.], p.32

[8.] See Source [1.], p.24

[9.] See Source [1.], p.13

[10.] See Source [5.], p.75

[11.] See Source [2.], p.46-55

[12.] Gimsa, A., The Proof of Mass Decay, International Journal of Scientific Research a. M., Volume 09, Issue 02, February 2021, DOI: 10.18535/ijsrm/v9i2.aa0, p.49

[13.] See Source [3.], p.12-13 